\title{
Livebirth after a Zona-Free ICSI of a Vitrified/ Warmed Oocyte after Social Freezing Running Title: Is it Worth to use Zona-Free Oocytes for ICSI?
}

\author{
Dunja Maria Baston-Büst*, Alexandra Petra Bielfeld, Nele Weber, Jan-Steffen Krüssel and Jens Hirchenhain \\ Department of OB/GYN, Düsseldorf University Hospital, Germany
}

Submission: February 20, 2019; Published: March 01, 2019

*Corresponding author: Dunja Maria Baston-Büst, Department of OB/GYN, Düsseldorf University Hospital, Germany

\begin{abstract}
Background: In the last decade, the number of female patients consulting fertility care units for egg freezing purposes increased. Reasons for oocyte banking for fertility preservation might be prevention of either premature ovarian insufficiency (e.g. due to cancer treatments, the so-called medical-freezing) or preventing the age-related fertility decline that is commonly termed "social egg freezing". The duration of oocyte storage depends on the initial age of the patient at oocyte retrieval, their social background, relationship status and civil status.
\end{abstract}

Case: We present one case of a zona-free ICSI after warming of oocytes that had been vitrified 4 years before (at patient's age 37) for oocyte banking. The oocyte left the zona in the last step of the warming procedure. ICSI was performed and the resulting pronuclear stage (2PN) was incubated in a time-lapse culture device up to blastocyst stage. The elective single ET of this blastocyst resulted in the livebirth of a healthy boy at 39 weeks of gestation.

Conclusion: ART units start to warm/thaw the oocytes of banking patients for non-medical indications. Patients consulting fertility care units for social egg freezing often differ from oocyte donors by their possibly advanced age and might therefore show a higher incidence of oocyte abnormalities. It is worth to inject zona-free oocytes and to observe them in a time-lapse device in order to observe a possible disaggregation of the blastomeres that might result in a multiple pregnancy if transferred in an earlier stage than morula or blastocyst.

Keywords: Oocyte banking; Embryo transfer; Time-lapse; ICSI, Social egg freezing; Vitrification; Warming; Fertility preservation

Abbreviations: ET: Embryo Transfer; ICSI: Intracytoplasmic Sperm Injection; POI: Premature Ovarian Insufficiency; ART: Assisted Reproductive Technique; MII: Metaphase II

\section{Introduction}

From the biological and medical point of view non-medical indicated oocyte freezing offers new perspectives especially for women between 25 and 33 years before the proportion of aneuploid oocytes rises dramatically [1]. Vitrification started to be applied in the daily labaratory routine worldwide since 2011 and is still often accompanied by the former slow freezing protocols [2]. This new technique is now the gold standard method in ART laboratories for mature oocytes [3,4]. The case presented herein describes a 37-year old patient, consulting the university's fertility care unit (UniKiD) in 2012. The patient decided to perform two cycles of controlled ovarian hyperstimulation and cryoprotection of the obtained MII oocytes for non-medical reasons. Interestingly, the patient was in a relationship in 2012, but denied to get pregnant at that time.

\section{Case Report}

Two cycles of ovarian hyperstimulation were performed with a long agonist protocol with recombinant follicle stimulating hormone (Puregon®, Merck Sharp \& Dohme B.V., BN Haarlem, Netherlands) combined with human menopausal gonadotropin (Menogon ®, Ferring International, Saint-Prex, Switzerland) within 3 months. Eight oocytes were cryopreserved in the first cycle ( 5 have been frozen with slow freezing (Sydney IVF Mature Oocyte Kit, Cook Medical, Limerick, Ireland) and 3 were vitrified

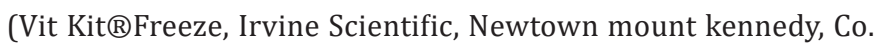
Wicklow, Ireland) using Cryotop @ devices (Kitazato, Tokyo, Japan).

12 oocytes were vitrified in the second cycle using the same media and devices. All procedures were performed according to manufacturer's standard protocols. This patient returned to our clinic 4 years later with the same male partner without occurrence of a naturally conceived pregnancy in between. For the frozen embryo transfer procedure, all the slow frozen oocytes from the first attempt and five vitrified oocytes from the second attempt were thawed/warmed (Vit Kit@Thaw, Irvine Scientific) and 7 of them survived and were injected by 
ICSI (Nikon Eclipse microscope, Nikon Instruments Europe BV, Amsterdam, Netherlands) with Eppendorf micromanipulation, Eppendorf AG, Hamburg, Germany) 4.5 hours after thawing/ warming. Prior to ICSI, 1 of the 5 oocytes that had been slow frozen and also 2 of the vitrified oocytes were degenerated. Sperm preparation and ICSI were performed according to the centres' standards. 2PN check was performed $19 \mathrm{~h}$ after ICSI and embryo culture (Continuous Single Culture Complete Medium $®$, Irvine Scientific) was performed in an embryoscope® (Vitrolife, Göteborg, Sweden).

\section{Result}
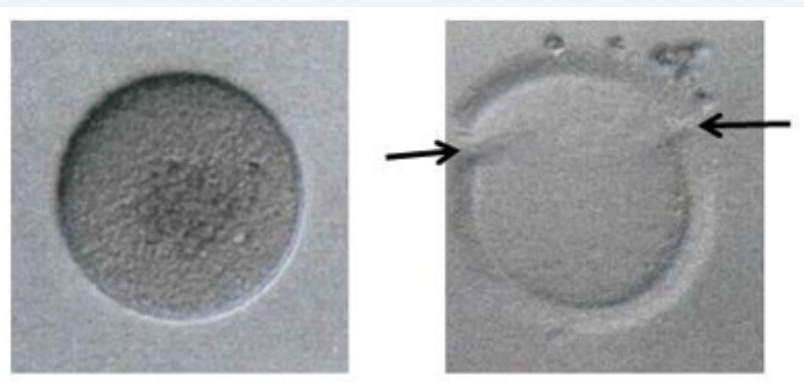

Figure 1: The zona-free oocyte and its zona after warming (200x magnification). Both sides with cracks are marked with an arrow.

Herein, we will focus on the fate of the zona-free oocyte that resulted from the vitrified cohort (Figure 1). The oocyte coincidentally left the zona in the last step of the warming procedure. The zona was photographed and 2 cracks were visible (Figure 1). The zona-free oocyte was handled with care and injected by ICSI together with the other oocytes. As 2PN were visible on the next morning, this $2 \mathrm{PN}$ was cultured in the embryoscope $®$ as well as the other 4 fertilized oocytes. Despite of an early reverse division (2-3-2 blastomeres), the embryo developed regularly and formed a blastocyst (grade 6AA) that was transferred to the patient in an artificial cycle with estrogen supplement (Estradot $100 \mu \mathrm{g}$, Novartis Pharma GmbH, Nürnberg, Germany) on day 6 of progesterone supplement (Crinone $\AA$, Merck, Darmstadt, Germany) (Supplement embryoscope ${ }^{\circledR}$ video). The patient reported 2 positive hCG blood tests ( 9 and 16 days after the transfer) and a clinical pregnancy approved by ultrasound. The patient delivered a healthy boy $(2790 \mathrm{~g}, 49 \mathrm{~cm})$ in the 39 th week of gestation by caesarean section.

\section{Discussion}

This is the first case-report of a livebirth after ICSI of an oocyte that turned out zona-free after warming of oocytes vitrified for non-medical reasons. Despite its function in protecting the oocyte from polyspermic and cross-species penetration, the zona supports contact between blastomeres in early embryonic development until compaction during morula stage. Generally, in most ART labs zona-free oocytes are discarded. The number of reports and case reports about ICSI of zona-free oocytes is limited [5]. Bodri et al. [6] reported about the zona-free ICSI procedure after removal of damaged zonas in 32 cases with ET of vitrified/warmed blastocysts. Already 20 years ago, Ding et al. tried to rescue one zona-free oocyte each in two couples with culturing up to blastocyst stage in order to avoid the physical and psychological burden of cycle cancellation for these patients [7].

Stanger et al. [8] treated a couple in two consecutive cycles with only zona-free oocytes. In the first cycle, the embryos arrested on day 2 , in the second attempt the corona cells had not been removed prior to the ICSI procedure and a clinical pregnancy occurred after a day 3 ET. In order to circumvent the loss of blastomere contact, the zona-free pronuclear stages or embryos could be retransferred in empty zonas [9]. The largest study on the morphology of zona-free oocytes was conducted by Ueno et al. with 97 zona-free oocytes compared with zona-intact oocytes [10].

They could not find any difference in neither fertilization rate nor cleavage and blastocyst formation rate.

Blastocysts were vitrified/warmed and led to the same clinical pregnancy rate, duration of gestation and no statistical differences regarding birthweight were observed [10]. Taken together, the damage of the zona might occur during follicular growth even in cases of natural cycle IVF or after ovum pickup during denudation. All reports available state the careful handling of zona-free oocytes during ICSI procedure and embryo culture. Time-lapse culturing simplifies the identification of any abnormalities during embryo development. Therefore, the use of zona-free oocytes should be considered especially in patients with a very low number of oocytes available. However, when choosing zona-free embryos for transfer it is mandatory to culture them to the blastocyst stage to prevent possible monozygotic multiples due to separation of single blastomeres before compaction occurs.

\section{References}

1. Hassold T, Hunt P (2009) Maternal age and chromosomally abnormal pregnancies: what we know and what we wish we knew. Curr Opin Pediatr 21(6): 703-708.

2. Levi-Setti, Patrizio P, Scaravelli G (2016) Evolution of human oocyte cryopreservation: slow freezing versus vitrification. Curr Opin Endocrinol Diabetes Obesity 23(6): 445-450.

3. Kuwayama M, Vajta G, Kato O, Leibo SP (2005) Highly efficient vitrification method for cryopreservation of human oocytes. Reprod Biomed Online 11(3): 300-308.

4. Cobo A, Meseguer M, Remohí J, Pellicer A (2010) Use of cryo-banked oocytes in an ovum donation programme: a prospective, randomized, controlled, clinical trial. Hum Reprod 25(9): 2239-2246.

5. Viganò $P$, Rubino P, Luddi A, Piomboni P (2015) The ICSI procedure from past to future: a systematic review of the more controversial aspects. Hum Reprod Update 22(2): 194-227.

6. Bodri D, Kato R, Kondo M, Hosomi N, Katsumata Y, et al. (2015) Timelapse monitoring of zona pellucida-free embryos obtained through in vitro fertilization: a retrospective case series. Fertil Steril 103(5): e35.

7. Ding J, Rana N, Dmowski W (1999) Intracytoplasmic sperm injection into zona-free human oocytes results in normal fertilization and blastocyst development. Hum Reprod 14(2): 476-478. 
8. Stanger J, Stevenson K, Lakmaker A, Woolcott R (2001) Pregnancy following fertilization of zona-free, coronal cell intact human ova: Case Report. Hum Reprod 16(1): 164-167.

9. Shu Y, Peng W, Zhang J (2010) Pregnancy and live birth following the transfer of vitrified-warmed blastocysts derived from zona- and corona-cell-free oocytes. Reprod Biomed Online 21(4): 527-532.

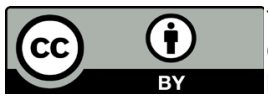

This work is licensed under Creative Commons Attribution 4.0 License

DOI: 10.19080/JGWH.2019.14.555887
10. Ueno S, Bodri D, Uchiyama K, Okimura T, Okuno T, et al. (2014) Developmental potential of zona pellucida-free oocytes obtained following mild in vitro fertilization. Fertil Steril 102(6): 1602-1607.

\section{Your next submission with Juniper Publishers will reach you the below assets}

- Quality Editorial service

- Swift Peer Review

- Reprints availability

- E-prints Service

- Manuscript Podcast for convenient understanding

- Global attainment for your research

- Manuscript accessibility in different formats

( Pdf, E-pub, Full Text, Audio)

- Unceasing customer service

Track the below URL for one-step submission https://juniperpublishers.com/online-submission.php 